\title{
Molecular mechanisms underlying attenuation of cisplatin-induced acute kidney injury by epicatechin gallate
}

\author{
Salma Malik,3, Kapil Suchal1,3, Jagriti Bhatia', Nanda Gamad' ${ }^{1}$, Amit Kumar Dinda², Yogendra Kumar Gupta ${ }^{1}$ and \\ Dharamvir Singh Arya'
}

Cisplatin, a platinum compound, is used as a first-line agent against various forms of solid cancers. Nephrotoxicity is an important adverse effect of cisplatin therapy, which involves increased oxidative stress, inflammation, apoptosis, and activation of the mitogen-activated protein kinase (MAPK) pathway. It is well known that the bioactive compounds present in green tea are used to treat various disorders due to their biological activities. With this background, the present study was aimed to investigate the effect of epicatechin gallate (ECG), a green tea polyphenol, in cisplatin-induced nephrotoxicity in rats. To achieve this, ECG $(1.25,2.5$, and $5 \mathrm{mg} / \mathrm{kg}$; intraperitoneal (i.p.)) was administered to male albino Wistar rats for the period of 10 days. On the 7th day, a single i.p. injection of cisplatin $(8 \mathrm{mg} / \mathrm{kg}$ ) was injected into rats to produce kidney injury and the animals were then killed on the 10th day. Cisplatin toxicity was associated with enhanced oxidative stress, impaired renal function along with marked tubular necrosis in Histopathology. Furthermore, cisplatin activated the MAPK pathway, which contributed to inflammation and apoptosis in the kidney of treated rats. In contrast, ECG $(5 \mathrm{mg} / \mathrm{kg})$ pretreatment normalized cisplatin-induced oxidative stress, renal function, and histopathological changes. ECG also prevented the activation of the MAPK pathway, and attenuated inflammation and apoptosis in rats. These findings suggest that ECG prevented cisplatin-induced oxidative stress, inflammation, and apoptosis by downregulating the MAPK pathway and resulted in improved renal function.

Laboratory Investigation (2016) 96, 853-861; doi:10.1038/labinvest.2016.60; published online 30 May 2016

Cisplatin (cis-diaminedichloroplatinum) is an effective chemotherapeutic agent widely used in the treatment of various epithelial malignancies such as ovarian, testicular, cervical, neck, and lung carcinoma. ${ }^{1}$ Despite its encouraging antitumor activity, its clinical usage is limited, because of dose-dependent and cumulative nephrotoxicity affecting $\sim 28-36 \%$ of patients receiving an initial dose of cisplatin $\left(50-100 \mathrm{mg} / \mathrm{m}^{2}\right) .^{2}$ Cisplatin induces apoptosis and necrosis of tubular epithelial cells predominantly in the inner cortex where it reaches its highest concentration. It selectively damages the S3 segment of proximal convoluted tubules, as this is the major site of excretion of cisplatin. ${ }^{3}$ Various treatment strategies such as saline hydration and diuresis have been suggested for prevention of cisplatin-induced nephrotoxicity. Induced diuresis may complicate the electrolyte imbalance caused by cisplatin, and at times, discontinuation of cisplatin remains the only option to prevent further tubular damage and morbidity. Presently, no specific pharmaceutical compound is approved to reverse renal damage, although such interventions are strongly required.

In tubular cells, cisplatin activates several stress signaling cascades such as the oxidative stress pathway, the tumor necrosis factor-alpha (TNF- $\alpha$ )-mediated inflammatory pathway, the mitogen-activated protein kinase (MAPK) signaling pathway, and various apoptotic pathways. ${ }^{1,2}$ In particular, MAPKs such as c-Jun $\mathrm{NH}_{2}$-terminal kinase (JNK), extracellular-regulated kinase (ERK) $1 / 2$, and p38 kinase are considered as important mediators of signal transduction processes that serve to regulate cell survival, cell division, gene expression, and cell metabolism in response to oxidative stress. ${ }^{4}$ They also function as upstream signals for TNF- $\alpha$-mediated inflammation and caspase-3-mediated

\footnotetext{
${ }^{1}$ Department of Pharmacology, Cardiovascular Research Laboratory, All India Institute of Medical Sciences, New Delhi, India and ${ }^{2}$ Department of Pathology, All India Institute of Medical Sciences, New Delhi, India

Correspondence: Dr J Bhatia, MBBS, MD, Department of Pharmacology, All India Institute of Medical Sciences, New Delhi 110029, India.

E-mail: jagriti2012@rediffmail.com

${ }^{3}$ These authors contributed equally to this work.

Received 8 September 2015; revised 18 March 2016; accepted 20 April 2016
} 
apoptosis in cisplatin-induced acute renal failure, and hence they are regarded as novel therapeutic targets in cisplatin nephrotoxicity. Various studies have shown that pharmacological modulation of these proteins can attenuate the toxic effects of cisplatin in kidney. ${ }^{5-9}$

Recent studies have revealed that green tea has healthprotecting benefits, especially in the prevention of diseases associated with free radicals and reactive oxygen species (ROS), such as cancer, cardiovascular, and neurodegenerative diseases. ${ }^{10-12}$ These beneficial effects are attributed to green tea catechins such as epicatechin gallate (ECG) and may be due to the presence gallate in the $3^{\prime}$ position. ${ }^{13}$ In addition to the antioxidant properties of ECG, various other bioactivities such as anti-diabetic, anti-bacterial, anti-inflammatory, and anti-obesity activities have been reported. ${ }^{14}$ Gallocatechins have also been asserted to influence molecular targets in signal transduction pathways associated with inflammation, cell death, and survival. ${ }^{15}$ In an in vitro study, ECG inhibited the production of pro-inflammatory cytokine, IL-1 $\beta$, and enhanced the production of anti-inflammatory cytokines, IL-10, in human leukocytes. ${ }^{16}$ Kim et al. ${ }^{17}$ showed that ECG attenuated ROS-induced oxidative stress in human nasal epithelial cells by inhibiting phosphorylation of ERK1/2 and epidermal growth factor receptor. However, it is not known whether ECG has nephroprotective effects, and if so, whether the molecular targets in signal transduction pathways are downstream events of the modulation of the pro-oxidant/ antioxidant balance in cells.

Therefore, the present study was performed to investigate possible nephroprotective effect of ECG in cisplatin-induced nephrotoxicity in rats by observing its effects on biochemical, morphological, apoptosis, and molecular parameters. Further, the molecular mechanism of action of ECG underlying its nephroprotective effect was also delineated.

\section{MATERIALS AND METHODS Reagents}

ECG and cisplatin were purchased from TCI Chemicals, India, and Pfizer Products, India, respectively. Blood urea nitrogen (BUN) and serum creatinine kits were obtained from Transasia Bio-Medicals, India. Terminal deoxynucleotide transferase dUTP nick-end labeling (TUNEL) assay kit (ApoBrduDNA fragmentation assay kit), TNF- $\alpha$, and interleukin-6 (IL-6) ELISA kits were obtained from Biovision, CA, Diaclone Tepnel Company, UK, and Ray Biotech, Norcross, GA, respectively. The antibodies against ERK1/2, phospho-ERK1/2 (p-ERK1/2), JNK, phospho-JNK (p-JNK), NF- $\kappa B p 65$, caspase- 3 , and $\beta$-actin were purchased from Cell Signaling Technology, USA. Phospho-p38 (p-p38) antibody was procured from Santa Cruz Biotechnology, USA. Antibodies specific for $\mathrm{Bcl}-2$, Bax, and p38 were obtained from Abcam, UK. Secondary anti-rabbit IgG and anti-mouse IgG antibodies were from Merck specialties, India. All other chemicals used were of analytical grade.

\section{Cisplatin-induced nephrotoxicity in rats}

A total of 36 adult male albino Wistar rats weighing between 150 and $200 \mathrm{~g}$ were used for the study. Rats were housed in polypropylene cages under standard laboratory conditions (temperature $\left(25 \pm 2^{\circ} \mathrm{C}\right)$ and relative humidity $(60 \pm 5 \%)$ ), and 12-h light/dark cycle with free access to standard diet (Ashirwad Industries, Chandigarh, India) and tap water ad libitum. All experimental procedures were approved by the Institutional Animal Ethics Committee of All India Institute of Medical Sciences, New Delhi, India (IAEC no. 675/12) and were performed according to the Indian National Science Academy Guidelines for Care and Use of Animals in Scientific research. Rats were divided into six groups (six rats in each group): normal; cisplatin-control; ECG treatment groups $(1.25,2.5$, and $5 \mathrm{mg} / \mathrm{kg}$; i.p.); and ECG per se group ( $5 \mathrm{mg} / \mathrm{kg}$; i.p.). ECG was dissolved in $0.5 \%$ dimethyl sulfoxide (DMSO) and administered at the dose of $1.25,2.5$, and $5 \mathrm{mg} / \mathrm{kg}$; i.p. for 10 days to rats in ECG treatment groups, whereas normal and cisplatin-control groups received $0.5 \%$ DMSO $(2 \mathrm{ml} / \mathrm{kg}$; i.p.) for the same time period and on the 7th day, animals in cisplatin and ECG treatment groups received single intraperitoneal injection of cisplatin $(8 \mathrm{mg} / \mathrm{kg})$. This dose $(8 \mathrm{mg} / \mathrm{kg})$ of cisplatin was selected on the basis of its efficacy in causing nephrotoxicity in rats. ${ }^{18}$

In all the groups, all the animals were anaesthetized with pentobarbitone sodium $(60 \mathrm{mg} / \mathrm{kg}$; i.p.) on the 10 th day of treatment. Blood was drawn from anaesthetized rats and centrifuged at 4000 r.p.m. to separate serum that was stored at $-20^{\circ} \mathrm{C}$ for estimating BUN, serum creatinine, TNF- $\alpha$, and IL-6 levels. Both the kidneys were dissected; one of the kidneys was immediately fixed in $10 \%$ neutral-buffered formalin, embedded in paraffin for histopathological, immunohistochemistry, and TUNEL assay. For biochemical estimation and western blot analysis, other kidney was snap-frozen in liquid nitrogen and stored at $-80^{\circ} \mathrm{C}$ until analysis.

\section{Measurement of Serum Nephrotoxic Markers}

Serum creatinine and BUN levels were measured by Jaffe's method and diacetylmonoxime method using respective commercially available kits.

\section{Measurement of Renal Biochemical Parameters}

For biochemical evaluation, kidney tissues were homogenized with 10 volumes of ice-chilled phosphate buffer $(0.1 \mathrm{M}, \mathrm{pH}$ 7.4). This homogenate was divided into two parts. One part of the homogenate was used for the estimation of malondialdehyde (MDA) level and reduced glutathione (GSH) content. ${ }^{19,20}$ Other part of the tissue homogenate was centrifuged at 4000 r.p.m., and the supernatant thus obtained was used for the estimation of superoxide dismutase (SOD), catalase (CAT) enzyme activities, and protein content. $^{21-23}$ 


\section{Determination of Serum TNF- $a$ and IL- 6 Level}

As per manufacturer's instructions, serum TNF- $\alpha$ and IL-6 levels were assessed using respective ELISA kits.

\section{Histopathological Evaluation of Renal Damage}

Paraffin blocks were cut into 5 - $\mu \mathrm{m}$-thick sections with microtome (Leica RM 2125, Germany). The sections were stained with hematoxylin and eosin and examined under light microscope at $\times 20$ magnification (Dewinter Optical, India).

\section{Immunohistochemical Studies to Assess Expression of Apoptotic and Inflammatory Markers}

Paraffin tissue blocks were cut into 5 - $\mu \mathrm{m}$ sections. They were deparaffinized and rehydrated by passing through xylene, graded series of ethanol, and water. Following this, antigen retrieval was performed by heating the sections in microwave oven at $95{ }^{\circ} \mathrm{C}$ for $10 \mathrm{~min}$ in citrate buffer $(10 \mathrm{mM}$; pH 6.0). Then sections were incubated in 30\% hydrogen peroxide in methanol for $10 \mathrm{~min}$ to reduce any endogenous peroxidase activity and with normal goat serum for $1 \mathrm{~h}$ at room temperature to block any non-specific binding. The tubular apoptosis and inflammatory cell infiltration was evaluated using antibodies such as Bax (1:500), Bcl-2 (1:500), caspase-3 (1:1000), and NF-кBp65 (1:1000) for $48 \mathrm{~h}$ that only react with antigens present in the cytoplasm of cells. The immune reaction was detected with horseradish peroxidase (HRP)-conjugated goat anti-rabbit IgG (1:3000) secondary antibody. The brown color was developed with 3,3'-diaminobenzidine (DAB). The sections were then counterstained with hematoxylin and mounted with DPX to visualize under light microscope (Dewinter Optical, India).

\section{TUNEL Assay}

TUNEL assay was performed to assess in situ detection of apoptosis in tissue sections. Tissue sections were incubated with Proteinase $\mathrm{K}$ for $30 \mathrm{~min}$ to enhance tissue permeability and then treated with $30 \% \mathrm{H}_{2} \mathrm{O}_{2}$ in methanol for $15 \mathrm{~min}$ to quench any endogenous peroxidase activity. After that, sections were incubated with complete labeling reaction buffer and antibody solution, each for $1 \mathrm{~h}$ and $30 \mathrm{~min}$. Colorimetric immune reaction was initiated by addition of DAB solution. At least five fields in each slide were looked for any TUNEL-positive cells in each group.

The pathologist examining histopathological, immunohistochemical, and TUNEL slides was blinded to the study protocol.

\section{Western Blot Analysis}

To elucidate the mechanism of action of its nephroprotective effect, modulation of protein expression was studied. Kidney tissue was removed from $-80^{\circ} \mathrm{C}$, weighed and homogenized in radioimmunoprecipitation assay lysis buffer, and total protein concentrations were measured using bovine serum album (BSA) as standard. Protein sample was separated in SDS-polyacrylamide gel electrophoresis and then transferred to a nitrocellulose membrane. Subsequently, membrane was blocked with 3\% BSA for $1 \mathrm{~h}$ and then incubated with primary antibodies for ERK1/2, p-ERK1/2, JNK, p-JNK, p38, p-p38, Bcl-2, Bax, caspase-3, NF- $\kappa B p 65$, and $\beta$-actin (1:3000) overnight at $4{ }^{\circ} \mathrm{C}$. The primary antibodies were detected with HRP-conjugated secondary antibodies (1:5000) at room temperature for $2 \mathrm{~h}$. Membrane-bound antibodies were visualized using enhanced chemiluminscence kit according to the manufacturer's instructions. The intensity of protein bands was determined with computerized densitometer.

\section{Statistical Analysis}

Data of various groups were analysed by one-way analysis of variance followed by post hoc Tukey-Kramer multiple comparison test using the InStat3.CNT software. Data are expressed as mean \pm s.e.m. Statistical significance was considered at the level of $P<0.05$.

\section{RESULTS}

\section{Effect of ECG on Renal Function}

Serum nephrotoxic markers such as serum creatinine and BUN levels were measured in all treatment groups to assess renal function. Cisplatin injection resulted in significant increases in serum creatinine $(P<0.001)$ and BUN levels $(P<0.001)$ in comparison with animals in the normal group. This elevation in serum nephrotoxic markers confirmed the induction of nephrotoxicity in the cisplatin-control group. ECG pretreatment $(5 \mathrm{mg} / \mathrm{kg})$, significantly normalized serum creatinine $(P<0.01)$, and BUN levels $(P<0.01)$, as compared with the cisplatin-control group (Figures $1 \mathrm{a}$ and $\mathrm{b}$ ).

\section{Effect of ECG on Cisplatin-Induced Oxidative Stress}

Rats injected with cisplatin showed a significant $(P<0.001)$ increase in the level of MDA, a lipid peroxidation marker, and a significant decrease in the activities of SOD $(P<0.001)$ and CAT $(P<0.01)$, as compared with the normal group (Figures $1 \mathrm{c}-\mathrm{e})$. Pretreatment with ECG $(5 \mathrm{mg} / \mathrm{kg})$ significantly $(P<0.05)$ decreased the level of MDA and a significant $(P<0.01)$ increase in the activities of SOD and CAT, as compared with the cisplatin-control group. In addition, there was significant depletion $(P<0.001)$ of GSH in the cisplatintreated group, as compared with the normal group. The kidneys of ECG pretreated rats showed a significant increase in the level of GSH when compared with the cisplatin-control group (Figure 1f).

\section{Effect of ECG on Serum Cytokine Levels}

The levels of serum pro-inflammatory cytokines (TNF- $\alpha$ and IL-6) were significantly enhanced $(P<0.001)$ in the cisplatincontrol group as compared to the normal group. This increased level of inflammatory cytokines in cisplatin-treated group indicates the role of inflammation in cisplatin-induced nephrotoxicity. ECG $(5 \mathrm{mg} / \mathrm{kg})$ pretreatment significantly $(P<0.01)$ attenuated the increase in cytokine level as compared to the cisplatin-control group. No significant 

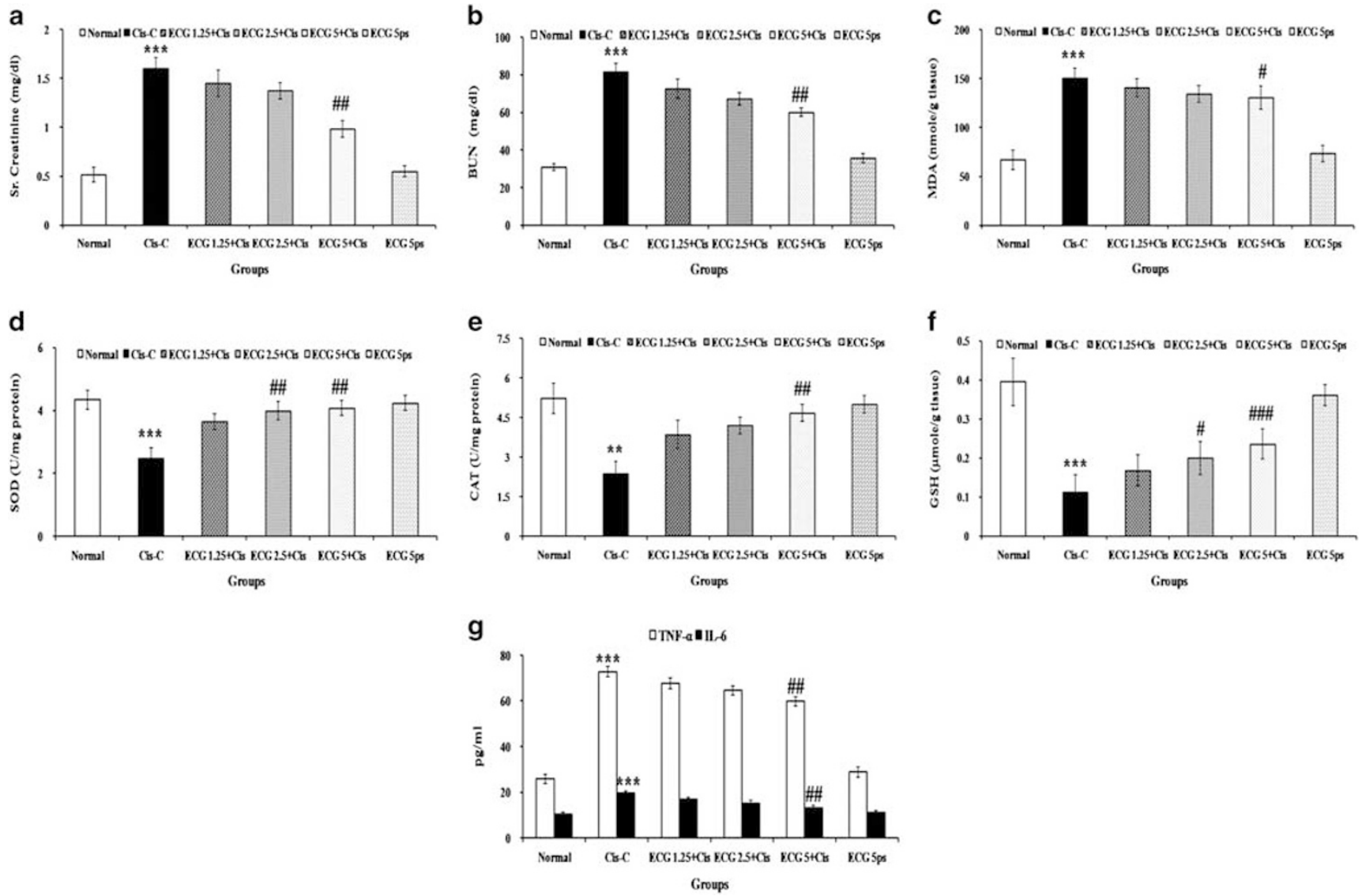

Figure 1 Effect of epicatechin gallate on (a) serum creatinine; (b) BUN; (c) MDA; (d) SOD; (e) CAT; (f) GSH; (g) TNF- $a$ and IL-6 levels following cisplatininduced acute kidney injury. Cis-C, cisplatin-control; ECG 1.25, 2.5, 5 mg/kg+Cis, epicatechin gallate 1.25, 2.5, 5 mg/kg+cisplatin; ECG 5 ps, epicatechin gallate $5 \mathrm{mg} / \mathrm{kg}$ per se. Data are expressed as mean \pm s.e.m. of six rats per group. BUN, blood urea nitrogen; CAT, catalase; GSH, reduced glutathione; IL-6, interleukin-6; MDA, malondialdehyde; SOD, superoxide dismutase; TNF- $a$, tumor necrosis factor- $a .{ }^{* *} P<0.01,{ }^{* * *} P<0.001$ vs normal group; ${ }^{\#} P<0.05$, ${ }^{\# \#} P<0.01,{ }^{\# \#} P<0.001$ vs Cis-C group.

differences were observed at lower doses (ECG 1.25 and $2.5 \mathrm{mg} / \mathrm{kg}$; Figure $1 \mathrm{~g})$.

\section{Effect of ECG on Renal Histology}

Histopathological sections exhibited normal architecture with no inflammatory cell infiltration in the normal and the ECG per se group, whereas the cisplatin-control group showed marked tubular atrophy, inflammation, and denudation of epithelium. Treatment with ECG ( 1.25 and $2.5 \mathrm{mg} / \mathrm{kg})$ reduced the cisplatin-induced changes of focal inflammation and necrosis. However, the group in which the rats received the highest dose of ECG $(5 \mathrm{mg} / \mathrm{kg})$, there was a marked absence of tubular necrosis and edema (Figure 2).

Thus, on the basis of serum kidney function, oxidative stress and histopathological evaluation, $5 \mathrm{mg} / \mathrm{kg}$ was found to be the optimum dose of ECG. Hence, this dose was used for further analysis.

\section{Effect of ECG on Apoptotic and Inflammatory Markers}

The apoptotic process was evaluated by detecting the expression of apoptotic the markers Bax, Bcl-2, and caspase-3 in renal tissue of all groups. Cisplatin administration increased expression of the pro-apoptotic proteins Bax and caspase- 3 , and decreased expression of the anti-apoptotic protein Bcl-2 in the renal tubules. In contrast, treatment with ECG attenuated apoptosis. ECG $(5 \mathrm{mg} / \mathrm{kg})$ pretreatment decreased the expression of Bax and caspase- 3 , and increased the expression of Bcl-2 in the renal tubules (Figures 3 and 4). Furthermore, apoptosis assessed using TUNEL assay showed decreased DNA fragmentation in ECG-treated rats compared with the cisplatin-control group. These findings confirm that ECG treatment prevents apoptosis in the renal tubules (Figure 3).

Immunohistochemical and western blot analysis also revealed increased expression of NF- $\mathrm{\kappa B}$, which is both proapoptotic and pro-inflammatory in the cisplatin-control group, whereas ECG pretreatment $(5 \mathrm{mg} / \mathrm{kg})$ ameliorated this effect (Figures 3 and 4).

\section{Effect of ECG on Phosphorylation of MAPKs}

The effect of ECG on protein expression was studied to explore its mechanistic pathway of renoprotection. Cisplatin 

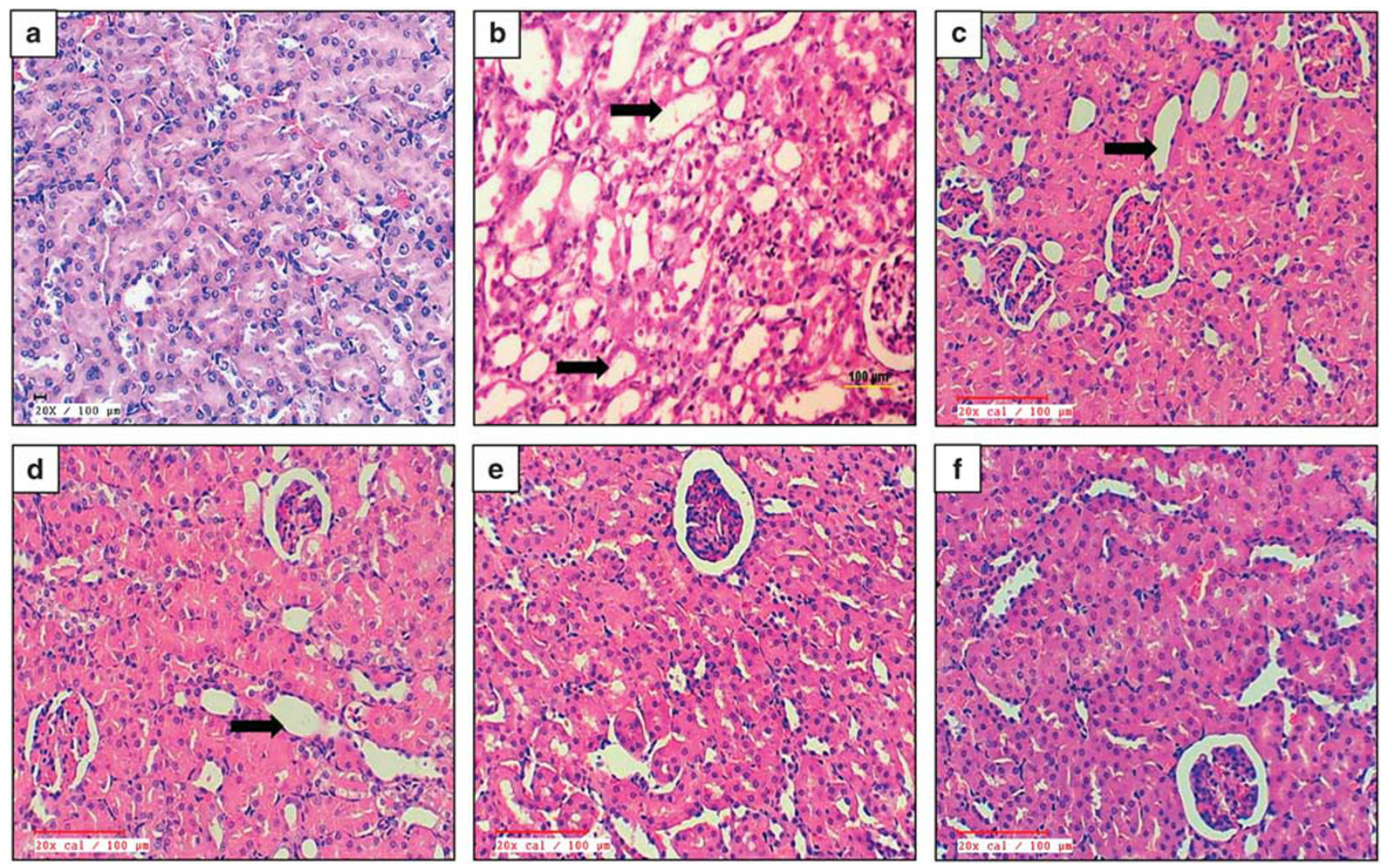

Figure 2 Effect of epicatechin gallate on morphological changes following cisplatin-induced acute kidney injury. (a) Normal; (b) Cis-C; (c) ECG 1.25 mg/ $\mathrm{kg}+\mathrm{Cis}$; (d) ECG $2.5 \mathrm{mg} / \mathrm{kg}+\mathrm{Cis}$; (e) ECG $5 \mathrm{mg} / \mathrm{kg}+\mathrm{Cis}$; (f) ECG 5 ps. Cis-C, cisplatin-control; ECG 1.25, 2.5, 5 mg/kg+Cis, epicatechin gallate 1.25, 2.5, 5 mg/ kg+cisplatin; ECG 5 ps, epicatechin gallate $5 \mathrm{mg} / \mathrm{kg}$ per se. $(\rightarrow)$ Acute tubular necrosis; $(n=3 ; \times 20$; scale bar, $100 \mu \mathrm{m})$.

injection resulted in increased phosphorylation of ERK1/2, JNK, and $\mathrm{p} 38$ in renal tissues. In contrast, treatment with ECG attenuated the activation of MAPK pathway (Figure 5).

\section{DISCUSSION}

Numerous studies have been conducted to enhance our understanding of the pathogenesis and treatment of cisplatininduced nephrotoxicity. In the present study, we have demonstrated the nephroprotective effect of ECG, a naturally occurring polyphenol in green tea in this condition. This is attributable to its capacity to elevate antioxidants, attenuate cisplatin-induced increased expression of MAPK, and the resultant inflammation and apoptosis/necrosis in the kidney associated with significant improvement in renal function.

For over a decade, both in vitro and in vivo studies have shown that oxidative stress has a critical role in the pathophysiology of cisplatin-induced cell death culminating in renal dysfunction. ${ }^{18,24,25}$ Inside the tubular cells, cisplatin is aquated into a highly reactive electrophile, which rapidly reacts with thiol-containing molecules including glutathione. It also induces mitochondrial dysfunction and increases ROS production by disrupting the respiratory chain. Because of their broad reactive nature, ROS targets and modifies lipids, proteins, and DNA resulting in cellular stress. ${ }^{1}$ Notably, ROS activates cascade of lipid peroxidation, which is detected by increased level of MDA (a lipid peroxidation marker) as seen in cisplatin-control group in our study. This probably resulted in consumption of glutathione and other endogenous antioxidants such as SOD and CAT. However, pretreatment with ECG maintained glutathione and other antioxidants at basal levels in renal tissue. Previous studies have shown that ECG can scavenge free radicals and convert them to non-toxic products. This action is attributed to the presence of galloyl group at position 3 and hydroxyl group at $5^{\prime}$ position. ${ }^{13}$ In addition, ECG treatment decreased the cisplatin-induced inflammatory response, and consequent cell death and renal failure. In cisplatin-treated rats, ECG markedly reduced the activation of NF- $\kappa B$, a transcription factor that activates pro-inflammatory cytokines TNF- $\alpha$ and IL-6 in response to oxidative stress. In human experiments, consumption of green tea extract, which contains ECG, led to reduced secretion of TNF- $\alpha .{ }^{15}$ Furthermore, a study by Siddique et al. $^{26}$ has demonstrated the protective role of ECG in a transgenic Drosophila model of Parkinson's disease due to its antioxidant and antiapoptotic effects. Tea polyphenols such as ECG have also been shown to inhibit proteasome function, thereby terminating inflammation. ${ }^{27}$ Hence, our data suggest that damage of renal tubules may be related to ROS-mediated 

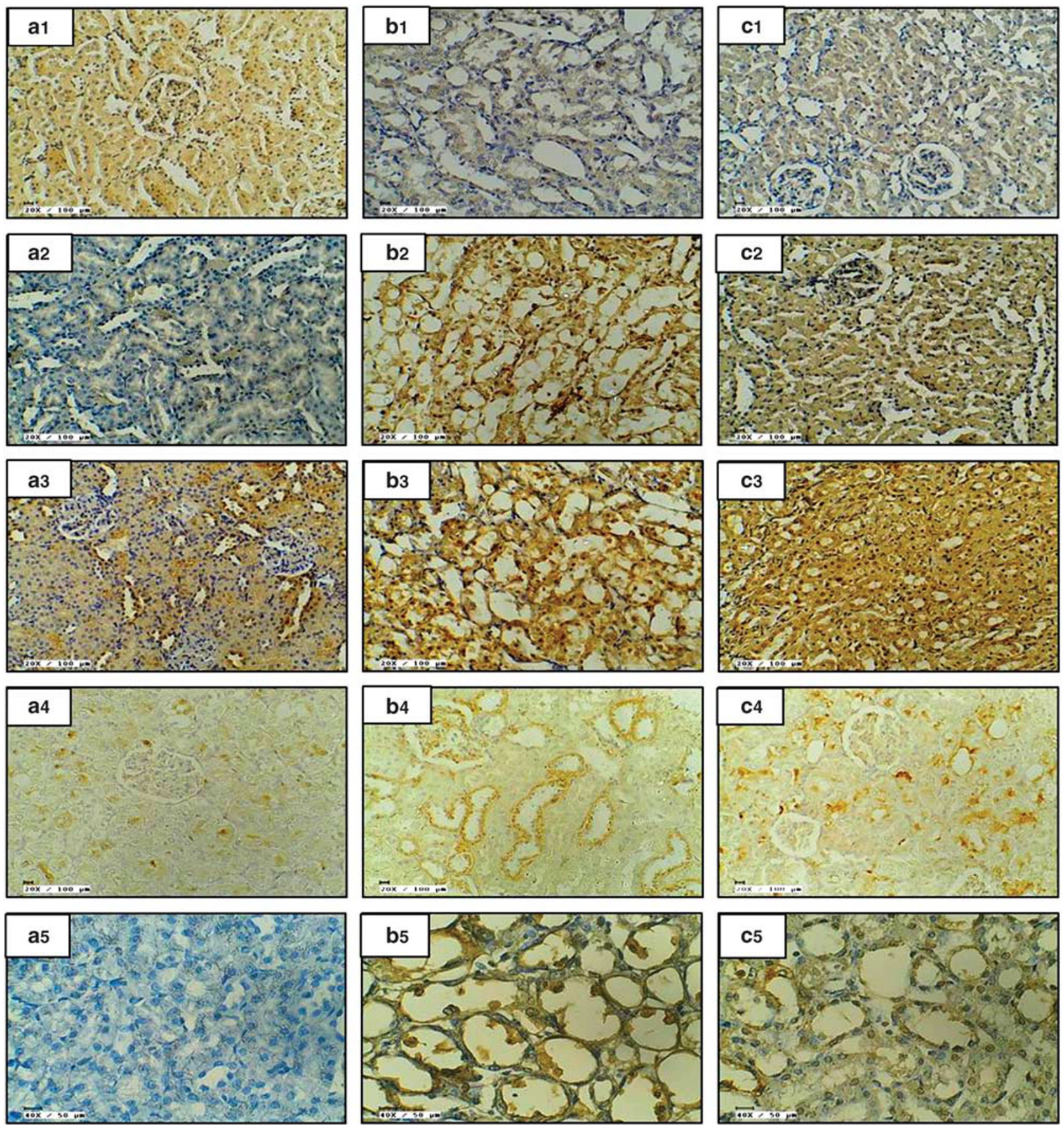

Figure 3 Effect of epicatechin gallate on Bcl-2 immunohistochemisty $(\mathbf{a} 1-\mathbf{c} 1 ; \times 20$; scale bar, $100 \mu \mathrm{m})$; Bax immunohistochemistry $(\mathbf{a 2}-\mathbf{c 2}$; $\times 20$; scale bar, $100 \mu \mathrm{m})$, NF-kBp65 immunohistochemistry (a3-c3; $\times 20$; scale bar, $100 \mu \mathrm{m})$, caspase-3 immunohistochemistry $(\mathbf{a} 4-\mathbf{c 4} ; \times 20$; scale bar, 100 $\mu \mathrm{m})$, and TUNEL positivity $(\mathbf{a} 5-\mathbf{c 5} ; \times$ 40; scale bar, 50 mm) following cisplatin-induced acute kidney injury. (a1-a5) Normal; (b1-b5) Cis-C; (c1-c5) ECG 5 mg/kg+Cis. Cis-C, cisplatin-control; ECG $5 \mathrm{mg} / \mathrm{kg}+\mathrm{Cis}$, epicatechin gallate $5 \mathrm{mg} / \mathrm{kg}+$ cisplatin.

cell inflammation and ECG may ameliorate cisplatin nephrotoxicity at these levels.

ECG also attenuated activation of the intrinsic mitochondrial pathway of apoptosis in renal tissue by decreasing the expression of $\mathrm{Bax}$ and caspase-3, and upregulating the antiapoptotic protein Bcl-2. The involvement of the intrinsic pathway of apoptosis in cisplatin-induced renal injury was initially found in cultured renal epithelial cells by Park et al. ${ }^{28}$ They provided evidence that accumulation of Bax following cisplatin treatment caused mitochondrial release of cytochrome $C$ and apoptosis. ${ }^{28}$ These initial observations were later tested and confirmed by several studies in different cell 
a
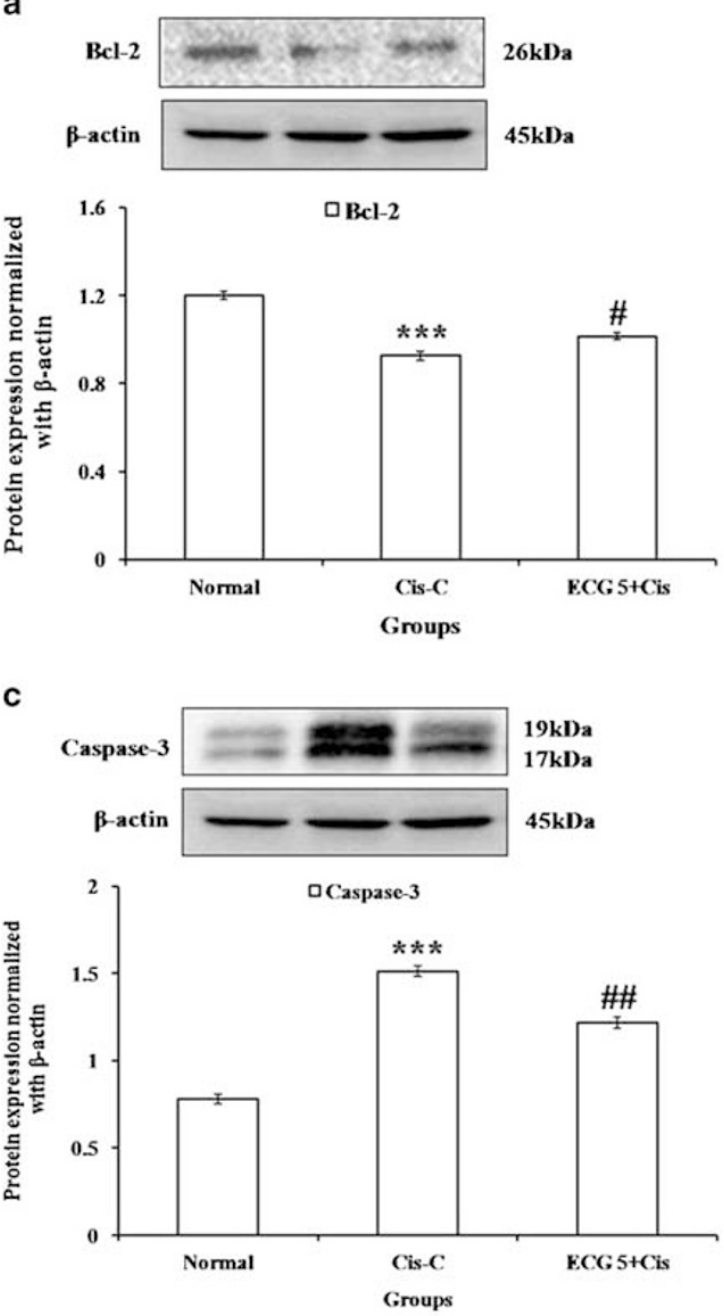

b
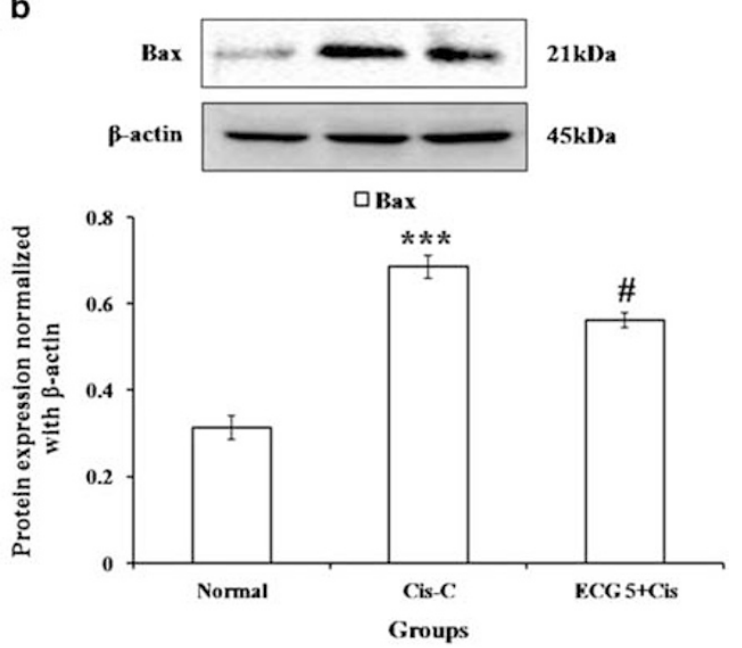

d

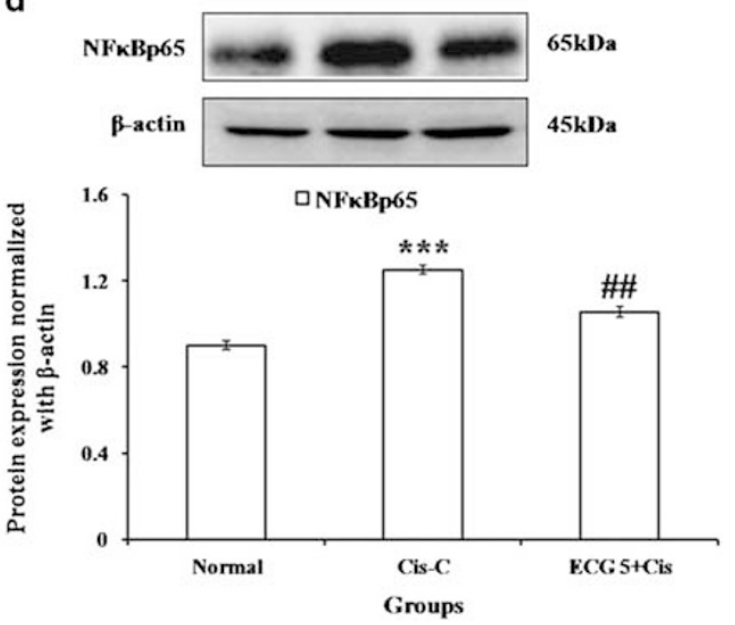

Figure 4 Effect of epicatechin gallate on various protein expression following cisplatin-induced acute kidney injury. (a) Bcl-2; (b) Bax; (c) caspase-3; (d) NF-KBp65. Data are expressed as mean \pm s.e.m. of three rats per group. ${ }^{* *} P<0.01,{ }^{* * *} P<0.001$ vs normal group; ${ }^{\#} P<0.05$, ${ }^{\# \#} P<0.01$ vs Cis-C group. Cis-C, cisplatin-control.

lines. Moreover, several pharmacological agents demonstrated anti-apoptotic effects by inhibiting Bax activation and accumulation in mitochondria. ${ }^{24,25,29}$ In addition, our present work has further demonstrated compelling evidence for tubular apoptosis via inhibition of formation of ROS and secretion of TNF- $\alpha$, which are known to activate the extrinsic pathway of apoptosis through death receptors. Alternatively, death receptors can generate ROS and enhance cisplatininduced acute kidney injury. Thus, oxidative stress and death receptor-mediated apoptosis appear to act synergistically. These molecular findings were further confirmed by histological examination and TUNEL assays. ECG pretreatment dramatically improved cisplatin-induced renal tubular damage and markedly reduced TUNEL-positive apoptotic cell numbers. Likewise, ECG pretreatment improved tubular function and normalized serum BUN and creatinine levels.

Although the exact mechanism of nephroprotective effect of ECG is not known, ECG certainly reduced the activation of MAPK by cisplatin. Studies have shown that cisplatin induces early ERK1/2, p38, and JNK/SAPK activation, which then precede the development of acute kidney injury and renal failure. ${ }^{30,31}$ Specific pharmacological inhibitors of ERK1/2, p38, and JNK reduced renal apoptosis and inflammation during cisplatin nephrotoxicity. Other studies further identified MAPK as key upstream regulators for the activation of TNF- $\alpha$ and caspase- 3 in response to oxidative stress. ${ }^{5-9}$ In light of these results, it is noteworthy that ECG decreased ROS activity and prevented downstream activation of MAPK/inflammation and apoptosis.

In conclusion, our study provides substantial evidence that ECG, a green tea polyphenol, may be of significant therapeutic benefit against the renal complications in cisplatin chemotherapy by maintaining redox potential. Specifically, ECG at $5 \mathrm{mg} / \mathrm{kg}$ had a promising therapeutic potential in ameliorating cisplatin-induced kidney injury. The putative 

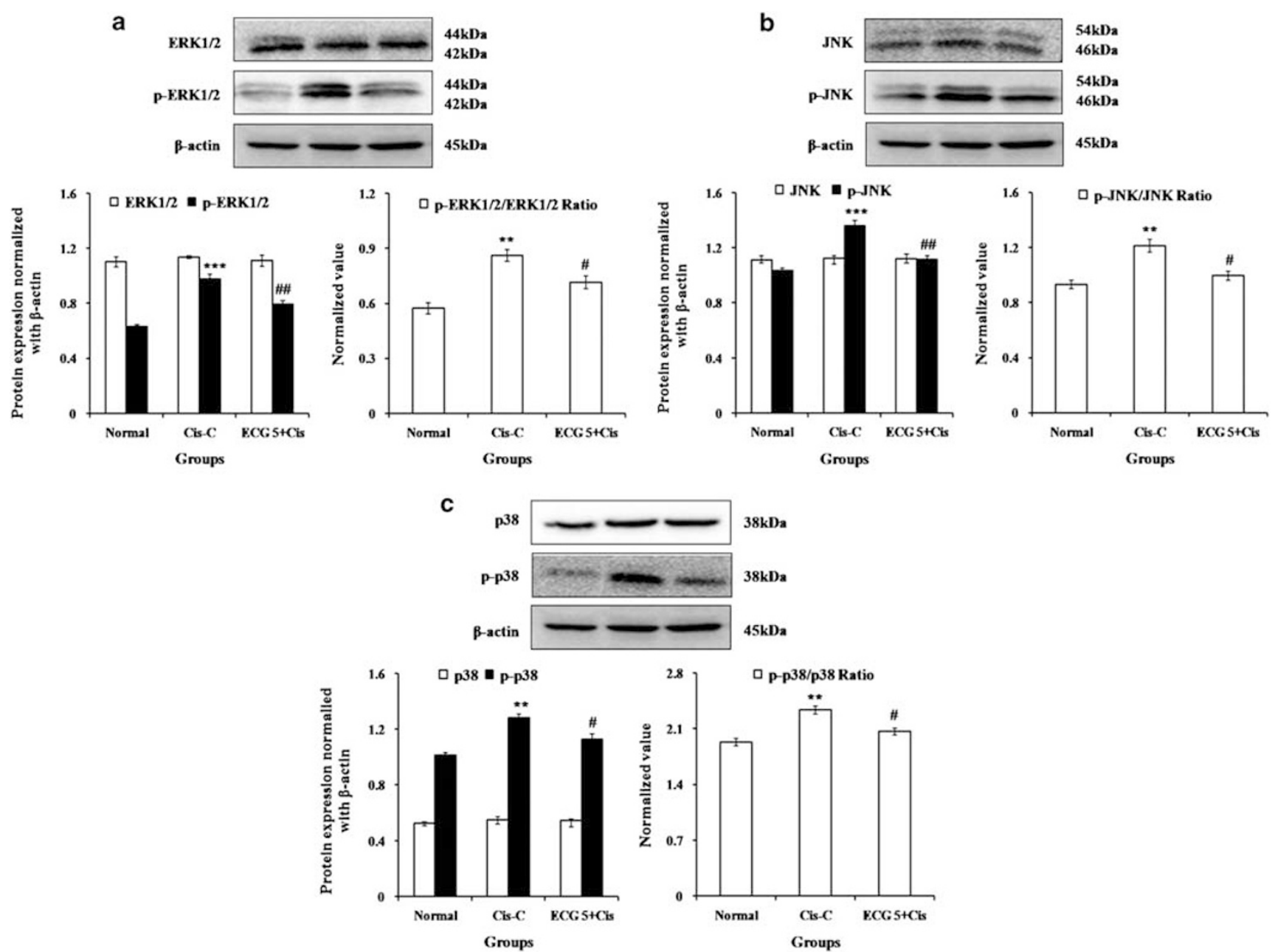

Figure 5 Effect of epicatechin gallate on MAPK phosphorylation following cisplatin-induced acute kidney injury. (a) ERK1/2 and p-ERK1/2; (b) JNK and p-JNK; (c) p38 and p-p38. Data are expressed as mean \pm s.e.m. of three rats per group. ${ }^{* *} P<0.01,{ }^{* * *} P<0.001$ vs normal group; ${ }^{\#} P<0.05$, ${ }^{\# \#} P<0.01$ vs Cis-C group. Cis-C, cisplatin-control; ERK1/2, extracellular-regulated kinase-1/2; JNK, c-Jun $\mathrm{NH}_{2}$-terminal kinase; MAPK, mitogen-activated protein kinase.

underlying mechanism of this renoprotective effect could be inhibition of MAPK-induced apoptosis and inflammation in cisplatin-injected renal tissues. However, further studies are necessary to establish the therapeutic usefulness of ECG to treat cisplatin-induced kidney damage.

\section{ACKNOWLEDGMENTS}

We are grateful to Mr B.M. Sharma for his technical assistance. Financial support was provided by Department of Biotechnology, Ministry of Science and Technology, New Delhi, India.

\section{DISCLOSURE/CONFLICT OF INTEREST}

The authors declare no conflict of interest.

1. Pabla N, Dong Z. Cisplatin nephrotoxicity: mechanisms and renoprotective strategies. Kidney Int 2008;73:994-1007.

2. Arany I, Safirstein RL. Cisplatin nephrotoxicity. Semin Nephrol 2003;23: 460-474.

3. Sahu BD, Rentam KK, Putcha UK, et al. Carnosic acid attenuates renal injury in an experimental model of rat cisplatin-induced nephrotoxicity. Food Chem Toxicol 2011;49:3090-3097.
4. Chang L, Karin M. Mammalian MAP kinase signalling cascades. Nature 2001:410:37-40.

5. Jo SK, Cho WY, Sung SA, et al. MEK inhibitor, U0126, attenuates cisplatin-induced renal injury by decreasing inflammation and apoptosis. Kidney Int 2005;67:458-466.

6. Francescato HD, Costa RS, Júnior FB, et al. Effect of JNK inhibition on cisplatin-induced renal damage. Nephrol Dial Transplant 2007;22: 2138-2148.

7. Luo J, Tsuji T, Yasuda $\mathrm{H}$, et al. The molecular mechanisms of the attenuation of cisplatin-induced acute renal failure by $\mathrm{N}$-acetylcysteine in rats. Nephrol Dial Transplant 2008;23:2198-2205.

8. Francescato HD, Costa RS, da Silva CG, et al. Treatment with a p38 MAPK inhibitor attenuates cisplatin nephrotoxicity starting after the beginning of renal damage. Life Sci 2009;84:590-597.

9. Kim HJ, Ravichandran K, Ozkok $A$, et al. The water-soluble riptolide derivative PG490-88 protects against cisplatin-induced acute kidney injury. J Pharmacol Exp Ther 2014;349:518-525.

10. Kinjo J, Nagao T, Tanaka T, et al. Activity-guided fractionation of green tea extract with antiproliferative activity against human stomach cancer cells. Biol Pharm Bull 2002;25:1238-1240.

11. Nie G, Jin C, Cao $Y$, et al. Distinct effects of tea catechins on 6-hydroxydopamine-induced apoptosis in PC12 cells. Arch Biochem Biophys 2002;397:84-90.

12. Yang YC, Lu FH, Wu JS, et al. The protective effect of habitual tea consumption on hypertension. Arch Intern Med 2004;164:1534-1540. 
13. Nakagawa T, Yokozawa T, Terasawa $\mathrm{K}$, et al. Protective activity of green tea against free radical- and glucose-mediated protein damage. J Agric Food Chem 2002;50:2418-2422.

14. Zaveri NT. Green tea and its polyphenolic catechins: medicinal uses in cancer and noncancer applications. Life Sci 2006;78: 2073-2080.

15. Murase T, Nagasawa A, Suzuki J, et al. Beneficial effects of tea catechins on diet-induced obesity: stimulation of lipid catabolism in the liver. Int J Obes Relat Metab Disord 2002;26:1459-1464.

16. Crouvezier S, Powell B, Keir D, et al. The effects of phenolic components of tea on the production of pro- and anti-inflammatory cytokines by human leukocytes in vitro. Cytokine 2001;13:280-286.

17. Kim HJ, Ryu JH, Kim CH, et al. Epicatechin gallate suppresses oxidative stress-induced MUC5AC overexpression by interaction with epidermal growth factor receptor. Am J Respir Cell Mol Biol 2010;43: 349-357.

18. Malik S, Suchal K, Gamad N, et al. Telmisartan ameliorates cisplatininduced nephrotoxicity by inhibiting MAPK mediated inflammation and apoptosis. Eur J Pharmacol 2015;748:54-60.

19. Ohkawa H, Ohishi N, Yagi K. Assay for lipid peroxides in animal tissues by thiobarbituric acid reaction. Anal Biochem 1979;95:351-358.

20. Moron MS, Depierre JW, Mannervik B. Levels of glutathione, glutathione reductase and glutathione S-transferase activities in rat lung and liver. Biochim Biophys Acta 1979;582:67-78.

21. Marklund $S$, Marklund $G$. Involvement of the superoxide anion radical in the autoxidation of pyrogallol and a convenient assay for superoxide dismutase. Eur J Biochem 1974;47:469-474.

22. Aebi H. Catalase in vitro. Methods Enzymol 1984;105:121-126.
23. Bradford MM. A rapid and sensitive method for the quantitation of microgram quantities of protein utilizing the principle of protein-dye binding. Anal Biochem 1976;72:248-254.

24. Kim TW, Kim YJ, Park SR, et al. Chrysanthemum indicum attenuates cisplatin-induced nephrotoxicity both in vivo and in vitro. Nat Prod Commun 2015;10:397-402.

25. Song KI, JY2 Park, Lee S3, et al. Protective effect of tetrahydrocurcumin against cisplatin-induced renal damage: in vitro and in vivo studies. Planta Med 2015;81:286-291.

26. Siddique $\mathrm{YH}$, Jyoti S, Naz F. Effect of epicatechin gallate dietary supplementation on transgenic Drosophila model of Parkinson's disease. J Diet Suppl 2014;11:121-130.

27. Yang H, Landis-Piwowar K, Chan TH, et al. Green tea polyphenols as proteasome inhibitors: implication in chemoprevention. Curr Cancer Drug Targets 2011;11:296-306.

28. Park MY, Lee $\mathrm{RH}$, Lee $\mathrm{SH}$, et al. Apoptosis induced by inhibition of contact with extracellular matrix in mouse collecting duct cells. Nephron 1999;83:341-351.

29. Gao S, Chen T, Choi MY, et al. Cyanidin reverses cisplatin-induced apoptosis in HK-2 proximal tubular cells through inhibition of ROSmediated DNA damage and modulation of the ERK and AKT pathways. Cancer Lett 2013;333:36-46.

30. Arany I, Megyesi JK, Kaneto $\mathrm{H}$, et al. Cisplatin-induced cell death is EGFR/src/ERK signaling dependent in mouse proximal tubule cells. Am J Physiol Renal Physiol 2004;287:F543-F549.

31. Kim JH, Park DJ, Yun JC, et al. Human adipose tissue-derived mesenchymal stem cells protect kidneys from cisplatin nephrotoxicity in rats. Am J Physiol Renal Physiol 2012;302:F1141-F1150. 